\title{
The Molecular Mechanism Regulating Diurnal Rhythm of Flavin-Containing Monooxygenase 5 in Mouse Liver ${ }^{\text {(s) }}$
}

\author{
Min Chen, ${ }^{1}$ Baozhang Guan, ${ }^{1}$ Haiman Xu, Fangjun Yu, Tianpeng Zhang, and Baojian Wu
}

Research Center for Biopharmaceutics and Pharmacokinetics (M.C., H.X., FY., T.Z., B.W.) and International Cooperative Laboratory of Traditional Chinese Medicine Modernization and Innovative Drug Development of Chinese Ministry of Education (MOE) (M.C., B.W.), College of Pharmacy, Jinan University, Guangzhou, China; and Department of Nephrology, the First Affiliated Hospital of Jinan University, Guangzhou, China (B.G.)

Received June 21, 2019; accepted August 28, 2019

\section{ABSTRACT}

Flavin-containing monooxygenase 5 (FMO5) is a phase lenzyme that plays an important role in xenobiotic metabolism. Here, we aimed to characterize diurnal rhythms of Fmo5 expression and activity in mouse liver and to investigate the potential roles of clock genes (Bmal1, Rev-erbo, and E4bp4) in the generation of diurnal rhythms. Fmo5 mRNA and protein showed robust diurnal rhythms, with peak values at zeitgeber time (ZT) 10/14 and trough values at ZT2/22 in mouse liver. Consistently, a diurnal rhythm was observed for in vitro microsomal Baeyer-Villiger oxidation of pentoxifylline (PTX), a specific reaction catalyzed by Fmo5. Pharmacokinetic studies revealed a more extensive Baeyer-Villiger oxidation of PTX at dosing time of ZT14 than at ZT2, consistent with the diurnal pattern of Fmo5 protein. Fmo5 expression was downregulated and its rhythm was blunted in $\mathrm{Bmal1}^{-/-}$and Rev-erbo ${ }^{-1-}$ mice. Positive regulation of Fmo5 by Bmal1 and Rev-erba was confirmed in primary mouse hepatocytes and/or Hepa1-6 cells. Furthermore, Fmo5 expression was upregulated and its rhythm was attenuated in E4bp4 ${ }^{-1-}$ mice. Negative regulation of Fmo5 by E4bp4 was validated using primary mouse hepatocytes. Combined luciferase reporter and chromatin immunoprecipitation assays demonstrated that Bmal1 (a known Rev-erb $\alpha$ activator) activated $F m 05$ transcription via direct binding to an E-box (-1822/-1816 bp) in the promoter, whereas E4bp4 (a known Rev-erb $\alpha$ target gene) inhibited Fmo5 transcription by binding to two D-boxes (-1726/-1718 and -804/-796 bp). In conclusion, circadian clock genes control diurnal expression of Fmo5 through transcriptional actions on E-box and D-box cis-elements.

\section{SIGNIFICANCE STATEMENT}

Hepatic Fmo5 displayed diurnal rhythmicities in expression and activity in mice. We uncovered the molecular mechanism by which the rhythmic Fmo5 expression was generated. Fmo5 promoter presents E-box and D-box binding elements for transcriptional actions from circadian clock proteins such as Bmal1, E4bp4, and Dbp. These findings have implications for understanding clockcontrolled drug metabolism and for facilitating the practice of chronotherapeutics.

\section{Introduction}

Flavin-containing monooxygenases (FMOs) are a family of phase I enzymes involved in metabolism of numerous drugs (e.g., benzydamine, methimazole, and albendazole) and environmental toxicants (e.g., insecticides, fonfos, and aldicarb) (Krueger and Williams, 2005; Rendic and Guengerich, 2015). There are five functional $F M O$ genes in humans, namely, FMO1, FMO2, FMO3, FMO4, and FMO5. The former four genes are clustered in the region 1q24.3 of chromosome 1, whereas

This work was supported by the National Natural Science Foundation of China (Grants 81722049 and 81573488), the Local Innovative and Research Teams Project of Guangdong Pearl River Talents Program (Grant 2017BT01Y036), the Natural Science Foundation of Guangdong Province (Grant 2017A03031387), the Guangzhou Science and Technology Project (Grant 201904010472), and the China Postdoctoral Science Foundation (Grant 2019M650234).

${ }^{1}$ M.C. and B.G. contributed equally to this work.

https://doi.org/10.1124/dmd.119.088450.

S This article has supplemental material available at dmd.aspetjournals.org.
FMO5 is located at $1 \mathrm{q} 21.1$. There is the same number of functional Fmo genes in mice. Fmo1, 2, 3, and 4 are clustered on chromosome 1 and Fmo5 is located on chromosome 3 (Hernandez et al., 2004). Of FMO genes, FMO5 is highly expressed in the liver in both humans and rodents (Janmohamed et al., 2004; Cashman and Zhang, 2006). A growing body of literature has revealed a critical role for human FMO5 in metabolism of drugs/metabolites, including pentoxifylline (PTX), nabumetone, E7016, and $S$-methyl-esonarimod (an active metabolite of the antirheumatic esonarimod) (Ohmi et al., 2003; Lai et al., 2011; Fiorentini et al., 2017). In addition, Fmo5 regulates metabolic aging via pleiotropic effects, including effects on fatty acid oxidation and biosynthesis of triglyceride, lipid, and cholesterol (Gonzalez Malagon et al., 2015).

PTX is a phosphodiesterase inhibitor used to treat peripheral vascular, inflammatory, and immune diseases (Samlaska and Winfield, 1994). PTX is mainly cleared from the body via phase I metabolism (Nicklasson et al., 2002; Raoul et al., 2007; Malátková and Wsól, 2014; Uney et al., 2019). Seven phase I metabolites (denoted M1-M7) can be generated from PTX in humans (Nicklasson et al., 2002). Recently,

ABBREVIATIONS: ANOVA, analysis of variance; AUC, area under the curve; Bmal1, brain and muscle arnt-like protein-1; ChIP, chromatin immunoprecipitation assay; Clock, circadian locomotor output cycles kaput; Cry, cryptochrome; Dbp, D-box binding protein; DMEM, Dulbecco's modified Eagle's medium; E4bp4, E4 promoter-binding protein 4; FBS, fetal bovine serum; FMO, flavin-containing monooxygenase; Fmo5-Luc, Fmo5 luciferase; HEK, human embryonic kidney; IBMX, 3-isobutyl-1-methyl xanthine; Ppib, peptidylprolyl isomerase B; Per, period; PTX, pentoxifylline; PTX-M, an oxidized metabolite of PTX; qPCR, quantitative reverse transcription-polymerase chain reaction; si, short-interfering; UPLC-QTOF/MS, ultra-performance liquid chromatography/quadrupole time-of-flight mass spectrometry; WT, wild-type; ZT, zeitgeber time. 
Fiorentini et al. (2017) identified a new metabolic pathway for PTX (i.e., Baeyer-Villiger oxidation) that is exclusively catalyzed by FMO5. This oxidation reaction may be used to probe the enzymatic activity of FMO5 (Fiorentini et al., 2017).

Many aspects of mammalian behaviors and physiology, such as sleep-wake cycles, heartbeat, blood pressure, and body temperature, are subject to circadian rhythms (Ralph et al., 1990; Gachon and Firsov, 2011). Circadian rhythms are driven by a central clock located in the suprachiasmatic nuclei of the hypothalamus and clocks present in peripheral tissues (Ralph et al., 1990; Ohdo et al., 2001; Gachon and Firsov, 2011). At the molecular level, the clock system consists of a set of clock genes/proteins [including brain and muscle Arnt-like protein-1 (BMAL1), circadian locomotor output cycles kaput (CLOCK), cryptochrome (CRY), and period (PER )] that operate using a transcriptionaltranslational feedback mechanism (Reppert and Weaver, 2002). BMAL1 and CLOCK proteins form a heterodimer to activate the transcription of $C R Y$ and PER and other clock-controlled genes (CCGs). When they reach a critical level, CRY and PER proteins inhibit BMAL1/CLOCK transactivation and downregulate their own expression and the expression of other CCGs (Gekakis et al., 1998; Kume et al., 1999). Thereby, the circadian clock generates oscillations in the expressions of CCGs.

Many drug-metabolizing enzymes (DMEs) including several P450s display circadian rhythms in their expressions (Zhang et al., 2009). Circadian expressions of DMEs are associated with dosing timedependent drug toxicity/tolerance and effects (Le'vi et al., 2010; Paschos et al., 2010). This knowledge may be exploited to optimize dosing regimen to minimize drug toxicity and maximize efficacy. Circadian clock genes appear to control the rhythmic expressions of DMEs using direct or indirect transcriptional mechanisms. For instance, Bmal1 regulates diurnal expression of Sult1a1 through direct binding to an E-box in the promoter (Guo et al., 2018). Rev-erb $\alpha$ regulates the circadian Ugt $2 b$ expression via binding to a RevRE element (Zhang et al., 2019). The PAR bZip (proline and acidic amino acid-rich basic-leucine zipper) transcription factors ( $D b p$, $H l f$, and Tef) control rhythmic expression of Cyp2b10 through transactivation of Car receptor (Paschos et al., 2010).

To date, the diurnal rhythmicities of FMOs (non-P450 phase I enzymes) remain unexplored. In this study, we aimed to characterize diurnal rhythms of Fmo5 expression and activity in mouse liver, and to investigate the potential roles of circadian clock genes [Bmal1, Rev$\operatorname{erb} \alpha$, and E4 promoter-binding protein 4 (E4bp4)] in generation of diurnal rhythms. We for the first time demonstrated that circadian clock genes control diurnal expression of Fmo5 through transcriptional actions on E-box and D-box cis-elements.

\section{Materials and Methods}

Materials. Pentoxifylline and 3-isobutyl-1-methyl xanthine (IBMX) were purchased from MCE (Monmouth Junction, NJ). Anti-Fmo5 (13699-1-AP) and anti-E4bp4 (11773-1-AP) antibodies were purchased from Proteintech (Chicago, IL). NADPH, anti-Rev-erb $\alpha$ (WH0009572M2) antibody, and Dulbecco's modified Eagle's medium (DMEM, high glucose) were purchased from SigmaAldrich (St. Louis, MO). Anti-Dbp (sc-32899) antibody was obtained from Santa Cruz Biotechnology (Santa Cruz, CA). Anti-Gapdh (ab181602) and anti-Bmal1 (ab3350) antibodies were obtained from Abcam (Cambridge, MA). The transfection reagent jetPRIME was purchased from Polyplus Transfection (Illkirch, France). Fetal bovine serum (FBS) and trypsin were obtained from Hyclone (Logan, UT). Short-interfering (si)RNA targeting Bmall, Rev-erb $\alpha$, and E4bp4 (named siBmall, siRev-erb $\alpha$, and siE4bp4, respectively; sequences provided in Table 1) were obtained from Transheep Bio-Tech (Shanghai, China). pRL-TK vector was purchased from Promega (Madison, WI).

Biosynthesis of PTX-M. PTX-M (an oxidized metabolite of PTX) was generated using human FMO5 supersomes (BD-Biosciences-Gentest, Woburn, MA) according to the microsomal metabolism assay protocol with a scaling factor of 10. Crude PTX-M (approx. $10 \mathrm{mg}$ ) was isolated and purified as described (Fiorentini et al., 2017). In brief, the microsomal reactions were performed in a centrifugal tube (total incubation volume: $2 \mathrm{ml}$ ) for 2 hours at $37^{\circ} \mathrm{C}$. The resulting mixture was extracted with ethyl acetate and the combined organic phases from 30 replicates were dried using Eppendorf Concentrator Plus (Hamburg, Germany). After reconstitution in dichloromethane, the crude extract was purified via chromatography on silica gel by using ethyl acetate/triethylamine (99:1, v/v), yielding about $2.0 \mathrm{mg}$ of PTX-M. The product was verified by ultraperformance liquid chromatography/quadrupole time-of-flight mass spectrometry (UPLC-QTOF/MS) analysis and showed an extract mass of $\mathrm{m} / \mathrm{z} 295.14$, andfragments of 252.13 and 181.08 (see Supplementary Fig. S1 for the fragmentation pathway).

Animal Studies. Male wild-type (WT) C57BL/6 mice were purchased from HFK Biotechnology (Beijing, China). Bmall ${ }^{-/}$(a C57BL/6 background) mice were obtained from Bioray Laboratories (Shanghai, China) (Guo et al., 2018). Rev-erbo ${ }^{-1-}$ (a C57BL/6 background) mice were obtained from Cyagen Biosciences (Guangzhou, China) (Wang et al., 2018). E4bp4 $4^{-1-}$ (a C57BL/6 background) mice were obtained from Dr. Masato Kubo (the Center for Integrative Medical Sciences, RIKEN, Japan) (Zhao et al., 2018). The control littermates of $\mathrm{Bmall}^{-/-}$, Rev-erb ${ }^{-/-}$, and $\mathrm{ESbp4}^{-/-}$mice are named Bmall ${ }^{+/+}$,

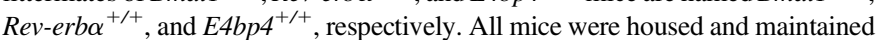
under a 12-hour light/12-hour dark cycle [lights on at 7:00 AM = zeitgeber time 0 (ZT0)] and lights off at 7:00 PM (= ZT12)], with food and water ad libitum at the Institute of Laboratory Animal Science (Jinan University, Guangzhou, China). Animal experiments were approved by Institutional Animal Care and Use Committee of Jinan University (Guangzhou, China). For diurnal expression profiling, liver tissues were collected from mice (male, $8-10$ weeks old, $n=5$ per group) every 4 hours over a 24-hour light-dark cycle at ZT2, ZT6, ZT10, ZT14, ZT18, and ZT22. The samples were subjected to quantitative reverse transcription-polymerase chain reaction (qPCR) and Western blotting assays.

For pharmacokinetic studies, PTX $(50 \mathrm{mg} / \mathrm{kg})$ was administered to WT mice (male, 8-10 weeks) by intraperitoneal injection (i.p.) at ZT2 or ZT14. At predetermined time points $(5,10,15,20,30,60,120$, and 240 minutes $)$, mice ( $n=$ 5 per time point) were rendered unconscious with isoflurane. Blood was sampled via a cardiac puncture and centrifuged at $8000 \mathrm{~g}$ for 8 minutes to collect the plasma. Livers were immediately collected, snap frozen in liquid nitrogen, and stored at $-80^{\circ} \mathrm{C}$. Frozen liver $(100 \mathrm{mg})$ was added two volumes of ice-cold saline, minced, and homogenized with IKA T25-Digital Ultra-Turrax (Staufen, Germany). Plasma and liver homogenates were mixed with methanol $(1: 10, v / v)$ (containing IBMX, an internal standard), vortexed for 3 minutes, and centrifuged at $13,000 \mathrm{~g}$ for 15 minutes. The supernatant was collected and dried using Eppendorf Concentrator Plus. The residue was reconstituted in $200 \mu \mathrm{l}$ of water/ methanol $(50: 50, \mathrm{v} / \mathrm{v})$, and centrifuged at $13,000 \mathrm{~g}$ for 15 minutes. The supernatant was injected into a UPLC-QTOF/MS system for drug and metabolite quantification (Waters, Milford, MA)

qPCR Assay. Total RNA was extracted from mouse liver or cell samples with TRIzol reagent (Invitrogen) following the manufacturer's instructions. cDNAs were synthesized from total RNA using a PrimeScript RT Master Mix (Takara Bio., Shiga, Japan). qPCR reactions were performed with GoTap qPCR Master Mix (Promegaa) using a Biometra Toptical Thermocycler (Analytik Jena, Goettingen, Germany) as described in our previous publication (Guo et al., 2018). The relative expression was determined using the $2^{-\Delta \Delta \mathrm{CT}}$ method. Peptidylprolyl isomerase B (Ppib) was used as an internal control. All primer sequences are provided in Table 1.

Western Blotting. Mouse tissues or cell samples were lysed in RIPA buffer (with $1 \mathrm{mM}$ phenylmethylsulfonyl fluoride). Protein concentrations were determined using a BCA assay kit (Beyotime, Shanghai, China). Protein samples $(40 \mu \mathrm{g})$ were subjected to $10 \%$ SDS-PAGE and transferred to a polyvinylidene fluoride membrane. The membrane was incubated with the primary antibody overnight at $4{ }^{\circ} \mathrm{C}$ after being blocked with 5\% nonfat milk in TBST for 1 hour, and then incubated with the secondary antibody for 1 hour at room temperature. Protein bands were visualized with enhanced chemiluminescence using an Omega LumG Imaging System (Aplegen, Pleasanton, CA) and analyzed by the Quantity One software. Protein levels were normalized to Gapdh and expressed as relative values.

Quantification of PTX and Its Metabolite. PTX and PTX-M were quantified using an UPLC-QTOF/MS system (Waters) and a BEH C18 column $(2.1 \times 50 \mathrm{~mm}$, $2.6 \mu \mathrm{m}$; Waters). The mobile phases were $0.1 \%$ formic acid in water (mobile 
TABLE 1

Oligonucleotides used in this study

\begin{tabular}{|c|c|c|}
\hline & Forward $\left(5^{\prime}-3^{\prime}\right.$ Sequence $)$ & Reverse $\left(5^{\prime}-3^{\prime}\right.$ Sequence $)$ \\
\hline \multicolumn{3}{|l|}{ qPCR } \\
\hline Bmal1 & CTCCAGGAGGCAAGAAGATTC & ATAGTCCAGTGGAAGGAATG \\
\hline $\operatorname{Rev}-\operatorname{erb} \alpha$ & TTTTTCGCCGGAGCATCCAA & ATCTCGGCAAGCATCCGTTG \\
\hline Fmo5 & GAGGGCTTGGAACCTGTCTG & CACGGACTGGTAAATACTGGC \\
\hline Ppib & TCCACACCCTTTTCCGGTCC & CAAAAGGAAGACGACGGAGC \\
\hline \multicolumn{3}{|r|}{ (1) } \\
\hline Fmo5-D-box ${ }^{1}$ & TGGCTTCCACTGCTATCC & TGAACCCAGGTCTTCTGC \\
\hline Fmo5-D-box ${ }^{3}$ & GCTTGCTTCCTGGGTTGG & GGTTCAGTATCTCCCTTCC \\
\hline Fmo5-distal & GGTAACCCAACGGCAACT & CTCACAGACATTCCCAAA \\
\hline \multicolumn{3}{|l|}{ siRNA } \\
\hline siBmal1 & GCUCUUUCUUCUGUAGAAUTT & AAAGAUAUCUUCCCUCGGTT \\
\hline siRev-erb $\alpha$ & UUCUCCGAACGUGUCACGUTT & ACGUGACACGUUCGGAGAATT \\
\hline siE4bp4 & GCACAAGCUUCGAUUAAATT & UUUAAUCCGAAGCUUGCTT \\
\hline
\end{tabular}

phase A) and $0.1 \%$ formic acid in acetonitrile (mobile phase B). The flow rate was set at $0.3 \mathrm{ml} / \mathrm{min}$. The gradient elution program was $10 \% \mathrm{~B}$ at $0-2$ minutes, $10 \%-90 \%$ B at $2-3.5$ minutes, $90 \%$ B at $3.5-4.5$ minutes, and $90 \%-10 \%$ B at $4.5-5$ minutes. The mass spectrometer was operated in positive ion mode. The capillary, sampling cone, and extraction cone voltages were set at 3000, 30, and $4 \mathrm{~V}$, respectively. The source and desolvation temperatures were 100 and $350^{\circ} \mathrm{C}$, respectively. Peak areas of PTX, PTX-M, and IBMX (internal standard) were recorded with extract masses of $m / z 279.15 \pm 0.05,295.14$ \pm 0.05 , and $223.01 \pm 0.05 \mathrm{Da}$, respectively. Representative extracted ion chromatograms are provided in Supplementary Fig. S1. The calibration curves for PTX and PTX-M were linear $\left(r^{2}>0.99\right)$ over the entire concentration range (i.e., 9.766-1250 ng/ml for PTX and 2-600 $\mathrm{ng} / \mathrm{ml}$ for PTX-M).

Plasmid Construction. Mouse Bmall (GenBank accession number: NM_007489.4), Rev-erb $\alpha$ (GenBank accession number: NM_145434.4),
A

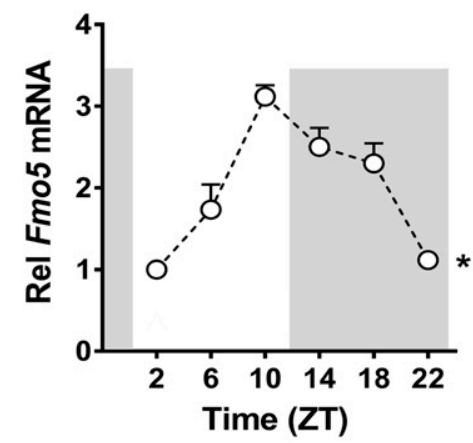

C<smiles>CC(=O)CCCCn1c(=O)c2c(ncn2C)n(C)c1=O</smiles>

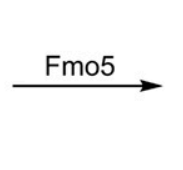

Pentoxifylline (PTX)

D

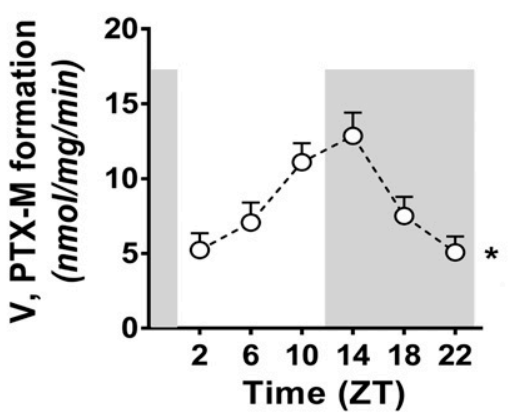

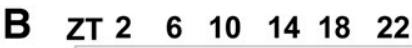
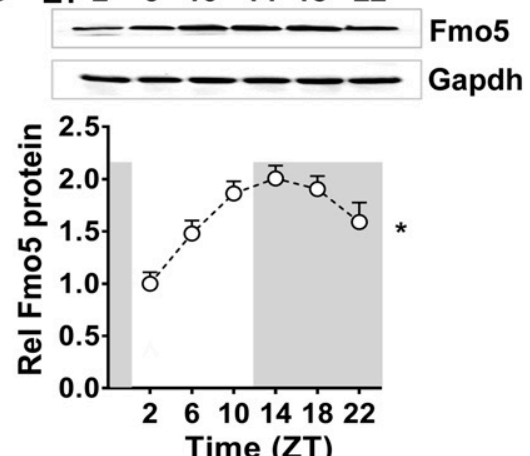

(ZT)<smiles>CC(=O)OCCCCn1c(=O)c2c(ncn2C)n(C)c1=O</smiles>

Oxidized metabolite (PTX-M)

E

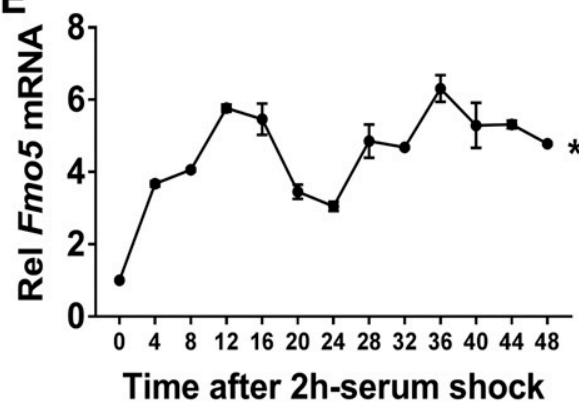

Fig. 1. Diurnal rhythm of Fmo5 expression in mouse liver and Hepa1-6 cells. (A) Diurnal rhythm of Fmo5 mRNA in mouse liver. Data are presented as the fold change in Fmo5 expression normalized to Ppib and relative to ZT2. (B) Rhythmic Fmo5 protein expression in mouse liver. Data are presented as the fold change in Fmo5 protein expression normalized to Gapdh and relative to ZT2. (C) Chemical structures of PTX and the Fmo5-generated metabolite PTX-M. (D) Diurnal rhythm of Fmo5 activity in mouse liver microsomes. (E) Temporal mRNA expression of Fmo5 in serumshocked Hepa1-6 cells. Data are presented as the fold change in Fmo5 expression normalized to Ppib and relative to time 0 . Data are mean \pm S.D. $(n=5) . * P<$ 0.05 (one-way ANOVA). The dark phase (lights off) is indicated in gray. Rel, Relative.V, metabolic rate. 


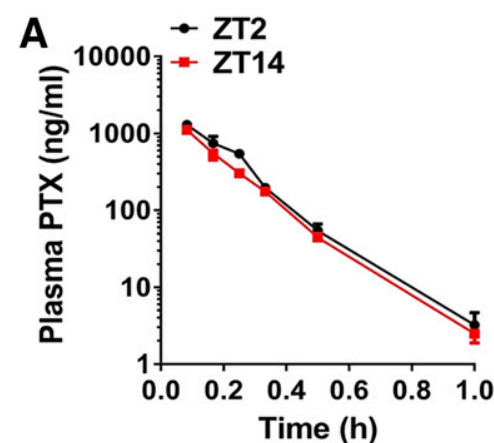

C

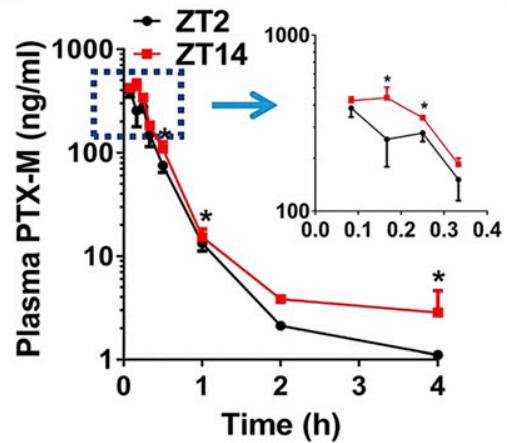

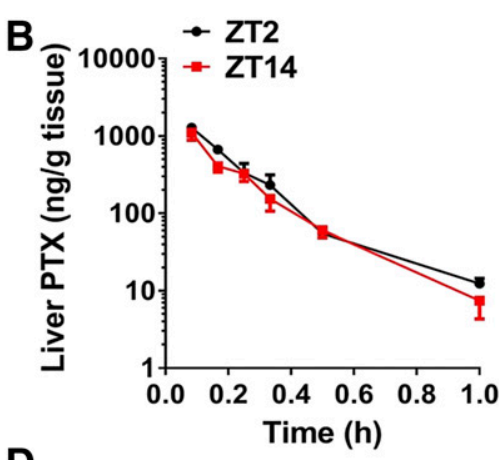

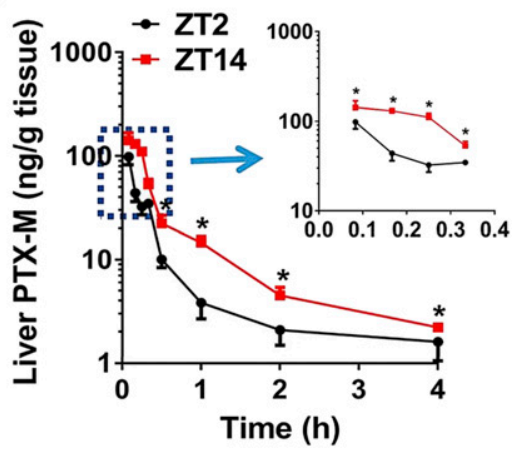

Fig. 2. Dosing time-dependent PTX-M formation from PTX in mice. (A) Plasma PTX concentration-time profile after dosing of PTX $(50 \mathrm{mg} / \mathrm{kg}$, i.p.) at ZT2 or ZT14. (B) Liver PTX concentration-time profile after dosing of PTX $(50 \mathrm{mg} / \mathrm{kg}$, i.p. $)$ at ZT2 or ZT14. (C) Plasma concentration-time profile of PTX-M after PTX administration $(50 \mathrm{mg} / \mathrm{kg}$, i.p.) at ZT2 and ZT14. (D) Liver concentration-time profile of PTX-M after PTX administration $(50 \mathrm{mg} / \mathrm{kg}$, i.p.) at ZT2 and ZT14. Data are presented as mean \pm S.D. $(n=5)$. $* P<0.05$ vs. ZT2 at individual time points (two-way ANOVA and Bonferroni post-hoc test).
E4bp4 (GenBank accession number: NM_017373.3), and Dbp genes (GenBank accession number: NM_016974.3) were synthesized and cloned into the expression vector pcDNA3.1 (Biowit Technologies, Shenzhen, China). Fmo5 luciferase (Fmo5-Luc) reporter constructs with different promoter fragments (i.e., $-2000 /+100,-1190 /+100,-910 /+100$, and $-600 /+100$ bp) and mutated versions were synthesized and cloned into the pGL4.17 vector (Kaile Bio-Tech, Guangzhou, China). The plasmids were transformed into Esherichia coli JM109 and extracted using an EasyPure HiPure Plasmid MiniPrep kit (TransGen Biotech, Beijing, China).

Isolation and Culture of Primary Mouse Hepatocytes. Primary hepatocytes were isolated from WT mice as previously described (Zhang et al., 2012, 2018a). In brief, mice were rendered unconscious with isoflurane. The liver was perfused with Hanks' balanced salt solution (HBSS) buffer through the portal vein and digested with collagenase IV. After being washed three times with HBSS buffer, hepatocytes were collected and cultured in DMEM supplemented with $10 \%$ FBS and $1 \%$ penicillin/streptomycin. Three hours later, the medium was changed to serum-free DMEM. On the next day, the cells were used for transfection.

Cell Transfection. Mouse hepatoma Hepa1-6 cells were purchased from the American Type Culture Collection (Rockville, MA). Cells were seeded into a sixwell plate and cultured in DMEM supplemented with $10 \%$ FBS at $37^{\circ} \mathrm{C}$ in a humidified $5 \% \mathrm{CO}_{2}$ atmosphere. Cells were transfected with Bmal1, Rev-erb $\alpha$, E4bp4 plasmid ( $2 \mu \mathrm{g}$ per well), siBmall, siRev-erb $\alpha, \operatorname{siE} 4 \mathrm{bp} 4(50 \mu \mathrm{M})$, or control using the jetPRIME transfection reagent (Polyplus Transfection). After a 48-hour transfection, cells were collected for qPCR and Western blotting analyses.

Serum Shock Experiments. Serum shock experiments were performed to induce circadian gene expression in Hepa1-6 cells as previously described (Matsunaga et al., 2012). In brief, Hepa1-6 cells were cultured in DMEM supplemented with $10 \%$ FBS. Once a confluence of $80 \%$ was reached, the culture medium was changed to serum-free DMEM. Twelve hours later, $50 \%$ FBS was added to the medium. After a 2-hour serum shock, the medium was changed back to serum-free DMEM, and the cells were collected at specific time points $(0,4,8,12,16,20,24,28,32,36,40,44$, and 48 hours) for qPCR assays.

Microsomal Metabolism Assay. Livers were collected from the WT mice every 4 hours over a 24-hour light-dark cycle at ZT2, ZT6, ZT10, ZT14, ZT18, and ZT22. Mouse liver microsomes were prepared by sequential ultracentrifugation, first at $9000 \mathrm{~g}$ for 10 minutes and then at $100,000 \mathrm{~g}$ for 1 hour (Zhang et al., 2018b). The protocol for microsomal incubation assay (in vitro Baeyer-Villiger oxidation reaction) was adapted from Lai et al. (2011). In brief, the incubation mixture (a total volume of $200 \mu \mathrm{l}$ ) contained $50 \mathrm{mM}$ potassium phosphate buffer (pH 7.4), $2 \mathrm{mg} / \mathrm{ml}$ liver microsomes, $2.5 \mathrm{mM} \mathrm{NADPH}$, and $500 \mu \mathrm{M}$ PTX. After a 2-hour incubation (under a linear condition) at $37^{\circ} \mathrm{C}$, the reaction was terminated by addition of $200 \mu \mathrm{l}$ ice-cold water/acetonitrile (50:50, v/v) (containing IBMX, an internal standard). The resulting mixture was centrifuged at $2000 \mathrm{~g}$ for

TABLE 2

Pharmacokinetic parameters derived fromPTX concentration-time profiles

PTX $(50 \mathrm{mg} / \mathrm{kg}$, i.p.) was administered to WT mice at ZT2 or ZT14.

\begin{tabular}{|c|c|c|c|}
\hline Parameter & Unit & ZT2 & ZT14 \\
\hline \multicolumn{4}{|l|}{ Liver } \\
\hline AUC & $\mathrm{ng} / \mathrm{ml} * \mathrm{~h}$ & $232 \pm 35.9$ & $219 \pm 24.5$ \\
\hline MRT & $\mathrm{h}$ & $0.30 \pm 0.04$ & $0.73 \pm 0.13 *$ \\
\hline $\mathrm{V}_{\mathrm{ss}}$ & $1 / \mathrm{kg}$ & $0.14 \pm 0.05$ & $0.41 \pm 0.16$ \\
\hline CL & 1/h per kilogram & $0.21 \pm 0.03$ & $0.22 \pm 0.03$ \\
\hline \multicolumn{4}{|l|}{ Plasma } \\
\hline AUC & $\mathrm{ng} / \mathrm{ml}^{*} \mathrm{~h}$ & $232 \pm 40.8$ & $222 \pm 26.9$ \\
\hline MRT & $\mathrm{h}$ & $0.19 \pm 0.01$ & $0.19 \pm 0.01$ \\
\hline $\mathrm{V}_{\mathrm{ss}}$ & $1 / \mathrm{kg}$ & $0.04 \pm 0.01$ & $0.04 \pm 0.01$ \\
\hline $\mathrm{CL}$ & 1/h per kilogram & $0.23 \pm 0.04$ & $0.23 \pm 0.03$ \\
\hline
\end{tabular}

${ }^{*} P<0.05$ vs. ZT2. 
TABLE 3

Pharmacokinetic parameters derived from PTX-M concentration-time profiles

PTX (50 mg/kg, i.p.) was administered to WT mice at ZT2 or ZT14.

\begin{tabular}{clll}
\hline Parameter & \multicolumn{1}{c}{ Unit } & ZT2 & ZT14 \\
\hline Liver & & & \\
AUC & $\mathrm{ng} / \mathrm{ml} * \mathrm{~h}$ & $25.4 \pm 0.71$ & $54.8 \pm 2.5^{*}$ \\
MRT & $\mathrm{h}$ & $1.62 \pm 0.02$ & $0.50 \pm 0.03 *$ \\
$\mathrm{~V}_{\mathrm{ss}}$ & $1 / \mathrm{kg}$ & $3.75 \pm 1.46$ & $0.66 \pm 0.08^{*}$ \\
CL & $1 / \mathrm{h}$ per kilogram & $1.48 \pm 0.13$ & $1.12 \pm 0.23 *$ \\
Plasma & ng/ml*h & $115 \pm 13.0$ & $183 \pm 12.1 *$ \\
AUC & $\mathrm{h}$ & $0.43 \pm 0.01$ & $0.41 \pm 0.01 *$ \\
MRT & $1 / \mathrm{kg}$ & $0.26 \pm 0.01$ & $0.25 \pm 0.05$ \\
$\mathrm{~V}_{\text {ss }}$ & $1 / \mathrm{h}$ per kilogram & $0.32 \pm 0.01$ & $0.27 \pm 0.01 *$ \\
CL & & & \\
\hline
\end{tabular}

${ }^{*} P<0.05$ vs. ZT2.

10 minutes, and the supernatant was subjected to UPLC-QTOF/MS analysis as described in the section of Quantification of PTX and Its Metabolite. The calibration curve for PTX-M was linear $\left(r^{2}>0.99\right)$ over the entire concentration range (i.e., 31.3-2000 $\mathrm{nM}$ ).

Luciferase Reporter Assay. HEK293T cells were purchased from the American Type Culture Collection. HEK293T cells were seeded into 48-well plates. After 24 hours, cells were transfected with $200 \mathrm{ng}$ of Fmo5 luciferase (firefly) reporter plasmid, $10 \mathrm{ng}$ of pRL-TK vector (an internal control with renilla luciferase gene), and overexpression plasmid (Bmall, E4bp4, or Dbp plasmid). Different amounts $(100,200$, and $300 \mathrm{ng})$ of overexpression plasmids were tested.
Control experiments were performed with the blank plasmid pcDNA3.1. After a 24-hour transfection, cells were collected and the luciferase activities were measured using the Dual-Luciferase Reporter Assay system and GloMax 20/20 Luminometer (Promega).

Chromatin Immunoprecipitation Assay. Chromatin immunoprecipitation (ChIP) assays were performed using an Enzymatic Chromatin IP kit (Cell Signaling Technology, Beverly, MA) as previously described (Chen et al., 2019). In brief, mouse liver was fixed in $37 \%$ formaldehyde for 15 minutes at room temperature and the reaction was stopped by glycine. The nuclei were obtained from crosslinked samples with lysis buffers and digested with micrococcal
A

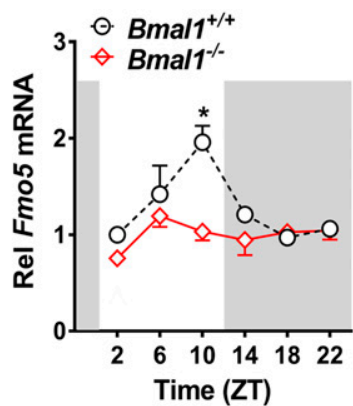

B

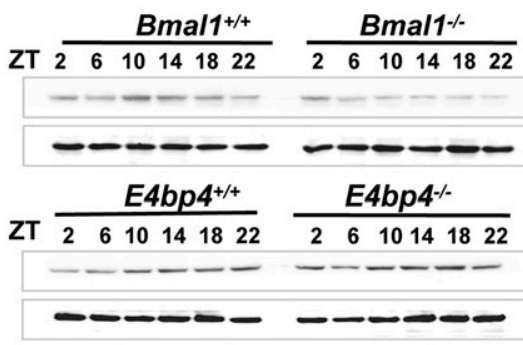

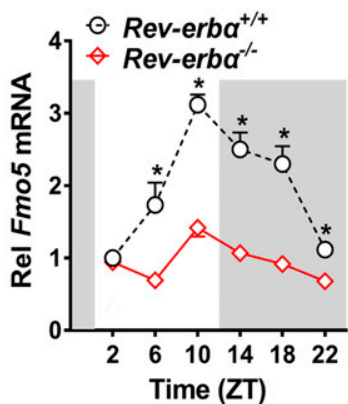

Time (ZT)

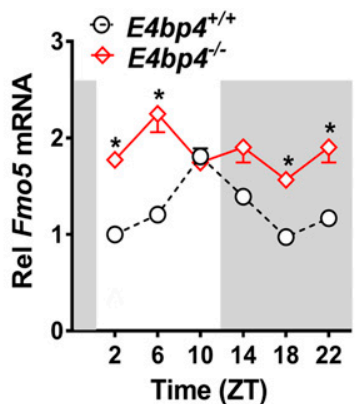

Time (ZT)

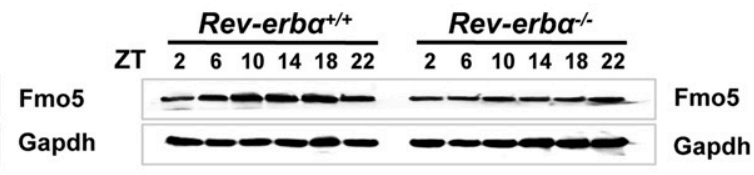

Fmo5

Gapdh
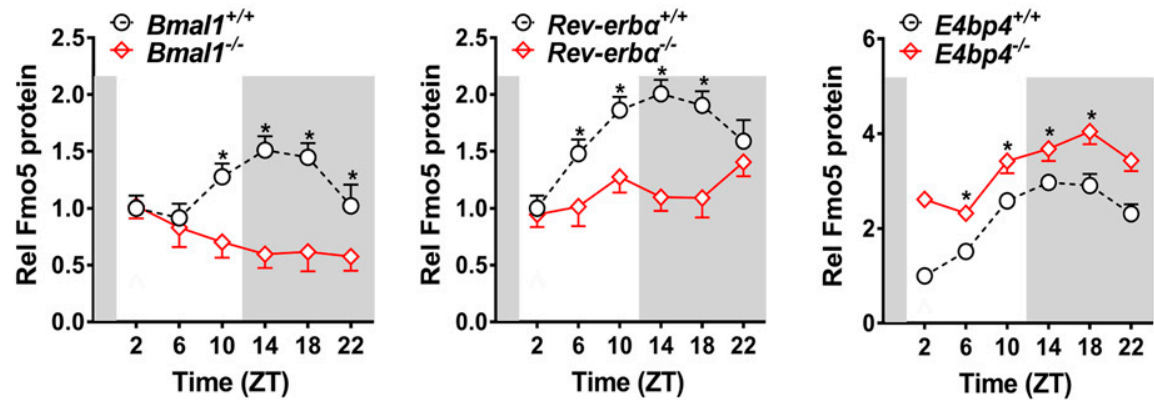

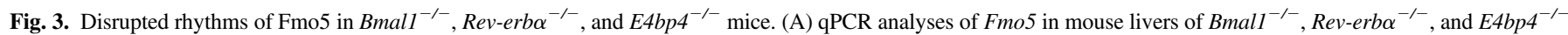
mice and their control littermates. Data are presented as the fold change in Fmo5 expression normalized to Ppib and relative to ZT2 of control littermates. (B) Western blotting analyses of Fmo5 protein in mouse liver of $\mathrm{Bmall}^{-/-}, \mathrm{Rev}_{\text {-erb }}{ }^{-/-}, E 4 b p 4^{-/-}$mice and their control littermates. Data are presented as the fold change in Fmo5 protein expression normalized to Gapdh and relative to ZT2 of control littermates. Data are mean \pm S.D. $(n=5) * P<0.05$ between different genotypes at individual time points (two-way ANOVA and Bonferroni post-hoc test). The dark phase (lights off) is indicated in gray. Rel, Relative. 
Primary mouse hepatocytes

A
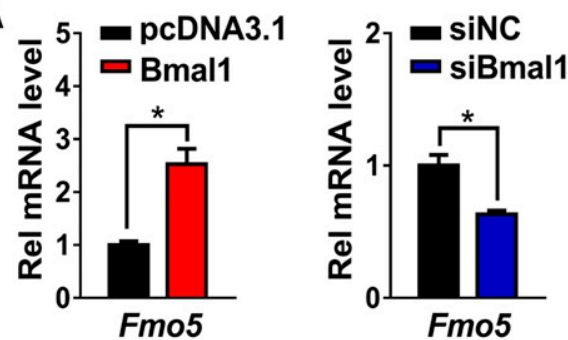

B
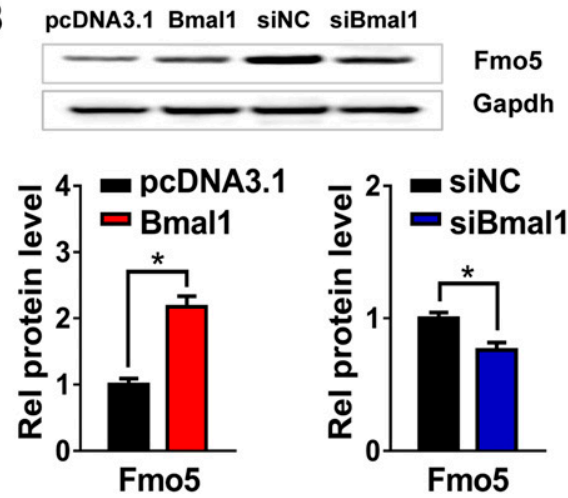

D

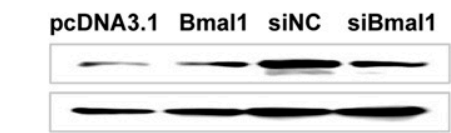

Gapdh
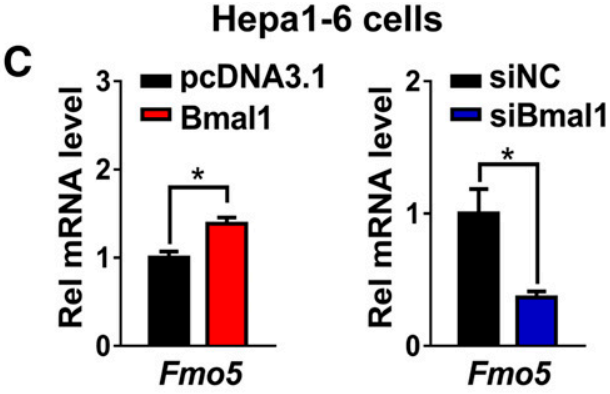

Fig. 4. Bmal1 regulates Fmo5 expression in primary mouse hepatocytes and Hepa1-6 cells. (A) Fmo5 mRNA expression in primary mouse hepatocytes transfected with Bmall expression plasmid or siBmal1. (B) Bmal1 induces Fmo5 protein expression in primary mouse hepatocytes. (C) Fmo5 mRNA expression in Hepa1-6 cells transfected with Bmall expression plasmid or siBmal1. (D) Bmal1 induces Fmo5 protein expression in Hepa1-6 cells. Data are mean \pm
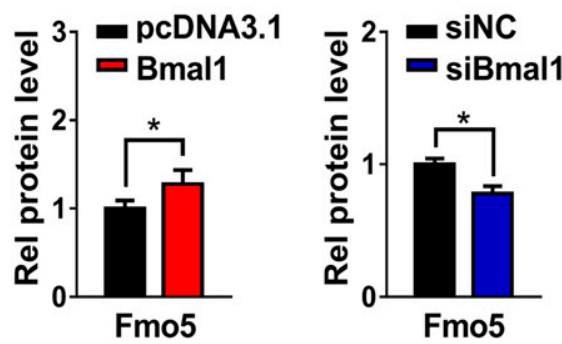
S.D. $(n=5)$. $* P<0.05$ ( $t$ test $)$. Rel, Relative. nuclease. The lysate was sonicated eight times ( 9 seconds each time) with an interval of 30 seconds to shear DNAs. The sheared chromatin (an average fragment size of $200 \mathrm{bp}$ ) was immunoprecipitated overnight with anti-Bmal1 (Abcam), anti-E4bp4 (Proteintech), anti-Dbp (Santa Cruz, CA), or normal rabbit IgG (control) at $4^{\circ} \mathrm{C}$. The chromatin was de-crosslinked at $65^{\circ} \mathrm{C}$ for 2 hours and purified with spin columns. The purified DNAs were analyzed by qPCR with specific primers (Table 1 ).

Statistical Analysis. Data are presented as means \pm S.D. Student's $t$ test was used to analyze the statistical differences between two groups. One-way or twoway analysis of variance (ANOVA) followed by Bonferroni post-hoc test was used for multiple group comparisons. The level of significance was set at $P<0.05$ (*).

\section{Results}

Diurnal Rhythms of Fmo5 Expression and Activity in Mouse Liver. Fmo5 mRNA in mouse liver oscillated in a circadian timedependent manner that peaked at around ZT10 (Fig. 1A). Likewise, Fmo5 protein showed a significant diurnal rhythm (Fig. 1B). Human FMO5 specifically catalyzes the formation of an oxidized metabolite (PTX-M) from PTX, also known as a Baeyer-Villiger oxidation reaction (Fig. 1C) (Fiorentini et al., 2017). We confirmed an exclusive activity of mouse Fmo5 in generating PTX-M from PTX (Supplementary Fig. S2). In vitro microsomal metabolism assays with PTX revealed a robust diurnal oscillation in Fmo5 activity consistent with the enzyme's rhythmic expression (Fig. 1D). We also observed rhythmic expression of Fmo5 in serum-shocked Hepa1-6 cells (Fig. 1E). These data supported Fmo5 as a circadian gene.

Circadian Time-Dependent In Vivo Activity of Fmo5. We next attempted to assess circadian time-dependent activity of Fmo5 in vivo by performing pharmacokinetic studies with PTX [dosed at a different circadian point (i.e., ZT2 or ZT14)]. Dosing time did not cause a significant difference in PTX pharmacokinetics, although ZT14 generated slight decreases in plasma and liver PTX concentrations compared with ZT2 (Fig. 2, A and B; Table 2). In contrast, a significant dosing-time effect was observed for PTX metabolism (i.e., formation of PTX-M). Both plasma and liver levels of PTX-M were higher at dosing time of ZT14 than at ZT2 (Fig. 2, C and D). Accordingly, higher area under the curve (AUC) values of PTX-M were observed for ZT14 (Table 3). The data indicated circadian time-dependent PTX-M formation in mice that was attributed to a diurnal rhythmicity in Fmo5 activity.

Disrupted Fmo5 Rhythms in Bmal1 ${ }^{-/-}$, Rev-erbo ${ }^{-/-}$, and $\boldsymbol{E A b p 4}^{-/-}$Mice. Circadian gene expression is generally produced by a transcriptional mechanism in which core clock genes act on three ciselements (E-box, D-box, and RevRE or RORE) in the target gene promoter (Minami et al., 2013). We thus first investigated the roles of the three cis-elements in generation of rhythmic Fmo5 expression using Bmal1 $^{-1-}, E 4 b p 4^{-1-}$, and Rev-erbo ${ }^{-1-}$ mice. Bmal1, E4bp4, and Rev$\operatorname{erb} \alpha$ are respective representative cis-acting proteins for E-box, D-box, and RevRE. The rhythms of Fmo5 mRNA and protein were blunted in Bmal1 $^{-/-}$, Rev-erbo ${ }^{-/-}$, and E4bp4 $4^{-/-}$mice compared with their littermates (Fig. 3). Fmo5 levels were generally downregulated in

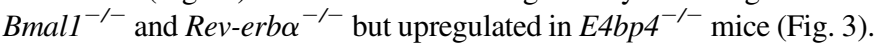
These data indicated that circadian clock genes Bmall, Rev-erb $\alpha$, and E4bp4 can modulate rhythmic Fmo5 expression probably through their own cis-elements.

Bmal1 Positively Regulates Fmo5 Expression in Cells. The regulatory effects of Bmal1 on Fmo5 expression were evaluated using primary mouse hepatocytes and Hepa1-6 cells. Overexpression of Bmal1 led to significant increases in Fmo5 mRNA and protein in primary mouse hepatocytes (Fig. 4, A and B). By contrast, knockdown of Bmal1 by siRNA caused decreases in Fmo5 mRNA and protein (Fig. 4, A and B). Likewise, Bmall overexpression resulted in increased Fmo5 expression in Hepa1-6 cells, whereas Bmal1 knockdown led to a reduction (Fig. 4, C and D). These data supported Bmal1 as a positive regulator of Fmo5.

E4bp4 and Rev-erba Regulate Fmo5 Expression in Primary Mouse Hepatocytes. The regulatory effects of E4bp4 and Rev-erb $\alpha$ on Fmo5 expression were assessed using primary mouse hepatocytes. Overexpression of E4bp4 caused significant decreases in Fmo5 mRNA and protein, whereas E4bp4 knockdown resulted in an increase in Fmo5 expression, revealing E4bp4 as a negative regulator of Fmo5 (Fig. 5, A and B). We observed positive regulatory effects of Rev-erb $\alpha$ on Fmo5 
A
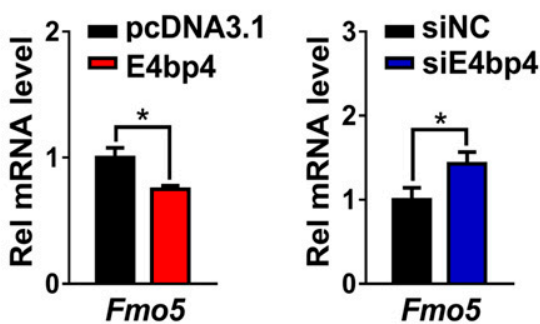

B
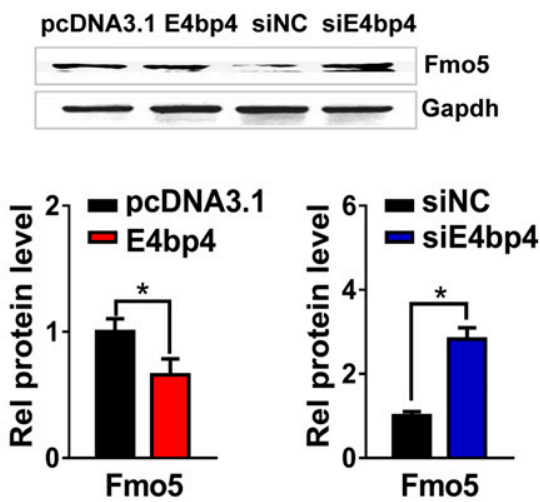

E

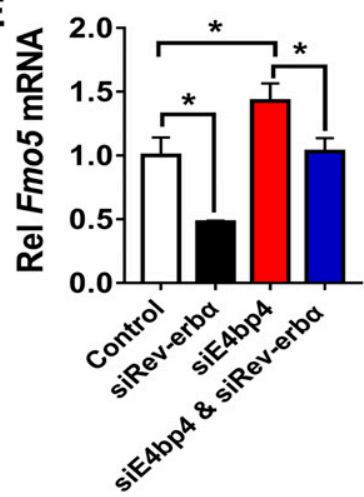

C

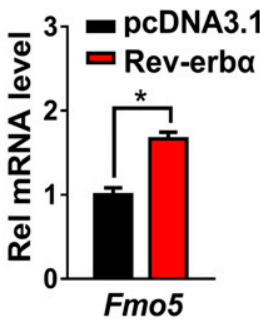

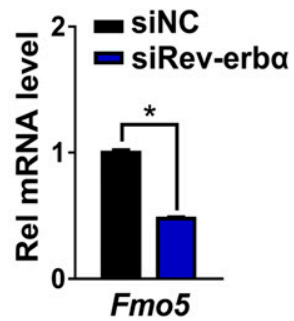

D
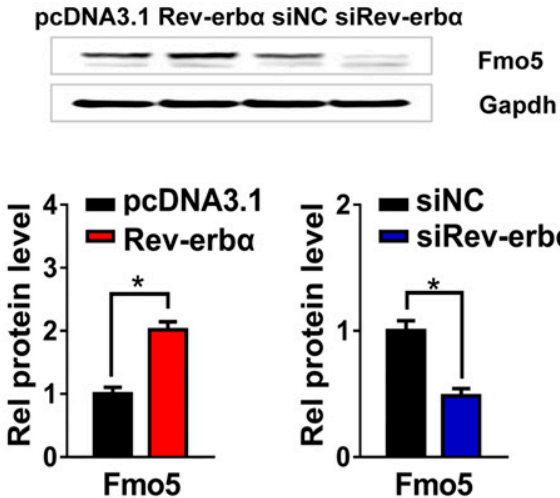

Fig. 5. E4bp4 and Rev-erb $\alpha$ regulate Fmo5 expression in primary mouse hepatocytes. (A) Effects of E4bp4 overexpression or knockdown on Fmo5 mRNA expression. (B) E4bp4 represses Fmo5 protein expression. (C) Effects of Rev-erb $\alpha$ overexpression or knockdown on Fmo5 mRNA expression. (D) Rev-erb $\alpha$ positively regulates Fmo5 protein expression. (E) Regulatory effects of Rev-erb $\alpha$ on Fmo5 are attenuated in E4bp4-deficient cells. Data are mean \pm S.D. $(n=5)$. $* P<0.05$ ( $t$ test $)$. Rel, Relative. (i.e., a positive correlation between Fmo5 and Rev-erb $\alpha$ expressions) (Fig. 5, C and D). This disfavored direct regulation of Fmo5 by Rev$\operatorname{erb} \alpha$ because Rev-erb $\alpha$ is a well known transcriptional repressor (Yin and Lazar, 2005). Instead, a negative mediator should be involved in positive regulation of Fmo5 by Rev-erb $\alpha$. Since E4bp4 is a known target gene of Rev-erb $\alpha$ (Duez et al., 2008), it was reasoned that positive regulation of Fmo5 by Rev-erb $\alpha$ was attained through transrepression of E4bp4, a negative regulator of Fmo5 (Fig. 5, C and D). This was supported by the fact that the regulatory effects of Rev-erb $\alpha$ on Fmo5 were attenuated in E4bp4-deficient cells (Fig. 5E).

Transcriptional Regulation of Fmo5 by Circadian Clock Genes. Computational algorithm (jaspar.genereg.net) predicted one potential E-box $\left(-1822 /-1816\right.$ bp) and three potential D-boxes (named D-box ${ }^{1}$, D-box $^{2}$, and D-box ${ }^{3}$ ) but no RevRE element in Fmo5 promoter (Fig. 6A). Bmal1 dose dependently induced Fmo5-Luc reporter $(-2000 /+100$ bp) activity (Fig. 6B). Truncation and mutation experiments demonstrated that -1816 bp E-box was essential for Bmal1 effect (Fig. 6C). A significant recruitment of Bmal1 protein to $-1816 \mathrm{bp} \mathrm{E-box} \mathrm{was} \mathrm{confirmed}$ using ChIP assays (Fig. 6D). These data indicated that Bmall directly transactivated Fmo5 through binding to an E-box in the gene promoter.

E4bp4 inhibited Fmo5-Luc reporter activity in a dose-dependent fashion, indicating a transcriptional repressive effect of E4bp4 on Fmo5
(Fig. 7A). Promoter analysis indicated that both D-box ${ }^{1}$ and D-box ${ }^{3}$ were essential for E4bp4 effect, whereas D-box ${ }^{2}$ made no contributions (Fig. 7B). ChIP assays confirmed binding of both D-box ${ }^{1}$ and D-box ${ }^{3}$ to E4bp4 protein (Fig. 7C). These data established E4bp4 as a direct repressor of Fmo5. D-box binding protein (Dbp) is a transcription factor reported to compete for the D-box to antagonize the transrepression effects of E4bp4 (Mitsui et al., 2001; Yu et al., 2019). In an identical manner, we found that Dbp transactivated Fmo5 through direct binding to D-box ${ }^{1}$ in the gene promoter (Fig. 7, D-F).

\section{Discussion}

In this study, we reported diurnal expression and activity of Fmo5 in mouse liver (Figs. 1 and 2). Moreover, we uncovered the molecular mechanism by which the rhythmic Fmo5 expression was generated (Fig. 8). To be specific, Fmo5 promoter presents E-box and D-box binding elements for transcriptional actions from circadian clock proteins such as Bmal1, E4bp4, and Dbp (Fig. 8). In addition to direct transactivation, the E-box binding protein Bmal1 may indirectly regulate the transcription of Fmo5 through Dbp and Rev-erb $\alpha / E 4 b p 4$ axis (Fig. 8). This is because $D b p$ and Rev-erb $\alpha$ are known target genes of Bmall and E4bp4 is a known target of Rev-erb $\alpha$ (Yamaguchi et al., 


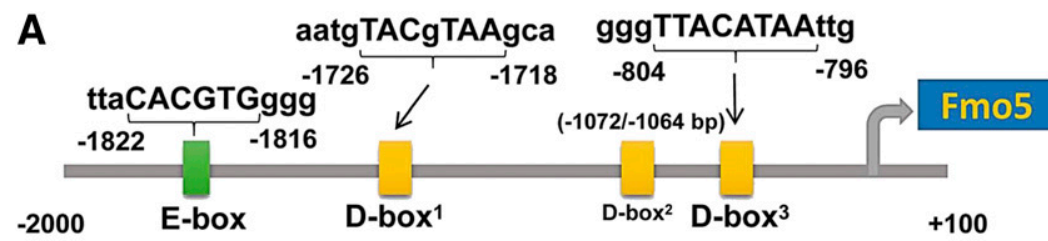

B

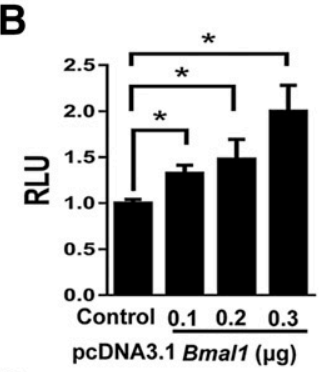

D

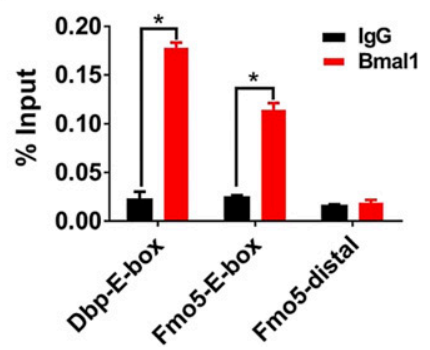

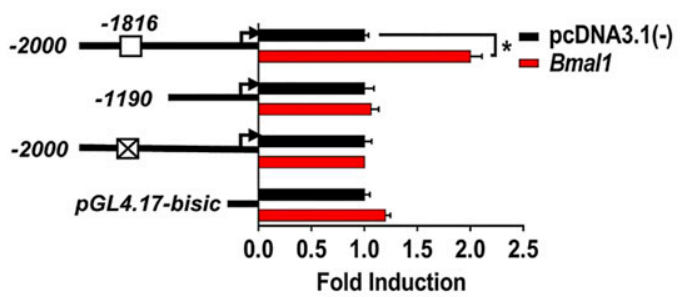

Fold Induction
Fig. 6. Bmal1 directly regulates Fmo5 expression through binding to an E-box element. (A) Schematic representation of E-box and D-box elements in Fmo5 promoter region. (B) HEK293T cell-based luciferase reporter assays. The cells were transfected with Fmo5Luc reporter (200 ng) and Bmall expression plasmid (100, 200, and $300 \mathrm{ng}$ ) or control. (C) HEK293T cell-based luciferase reporter assays. The cells were transfected with truncated or mutated versions of Fmo5 promoter (200 ng) and Bmall expression plasmid (300 ng). (D) ChIP assays showing recruitment of Bmal1 protein to Fmo5 promoter ( -1822 to -1816 bp region, an E-box element). An E-box of Dbp and a nonspecific (distal) sequence (Table 1) were used as positive and negative controls respectively. Data are mean \pm S.D. $(n=5)$. $* P<0.05$ ( $t$ test). RLU: relative luciferase unit.
2000; Mukherji et al., 2013; Yu et al., 2019). The indirect regulation mechanism was supported by the fact that hepatic Dbp and Rev-erb $\alpha$ were downregulated, whereas E4bp4 was upregulated in $\mathrm{Bmall}^{-/-}$mice (Supplementary Fig. S3).

Identifying circadian genes among drug-processing genes and elucidating the circadian regulation mechanisms are essential to advance chronopharmacology. Although a number of drug-processing genes are subjected to diurnal variations on the basis of an mRNA determination (Zhang et al., 2009), little is known regarding the circadian mechanisms regulating these genes. This is the first report revealing the molecular mechanisms controlling the diurnal expression of Fmo5, a non-P450 phase I enzyme. Since Fmo5 regulates metabolic aging via pleiotropic
A
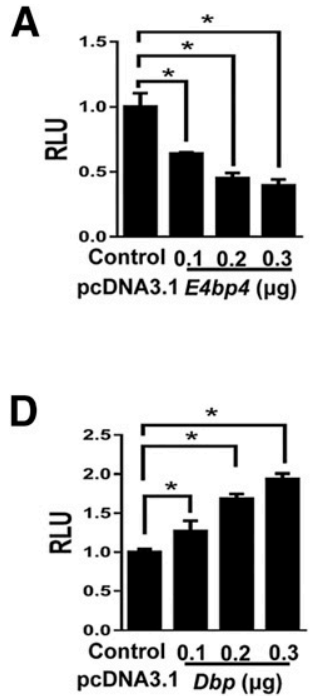

B

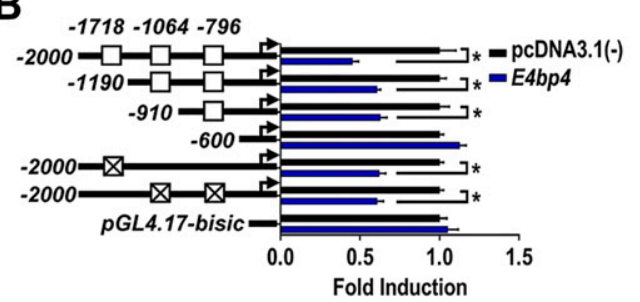

E

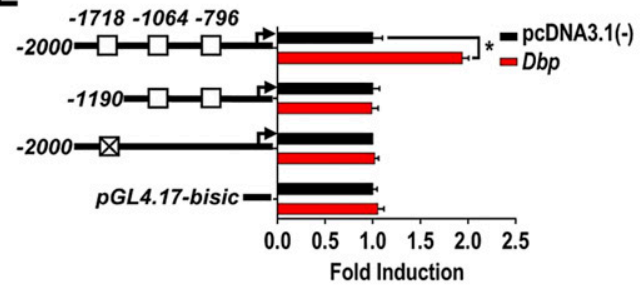

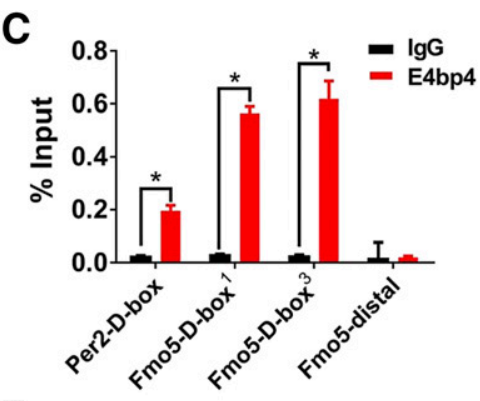

$\mathbf{F}$

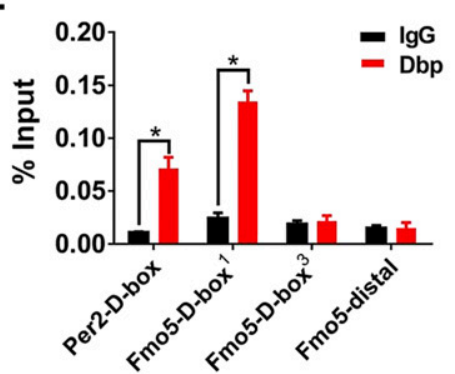

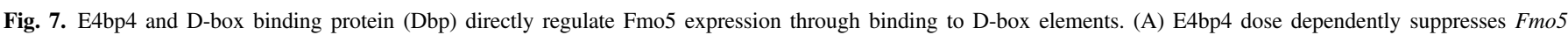

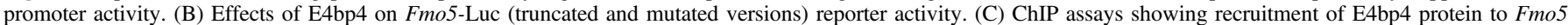

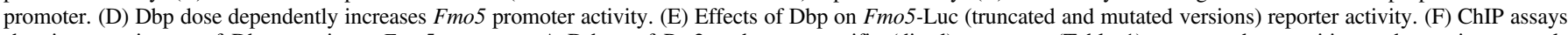

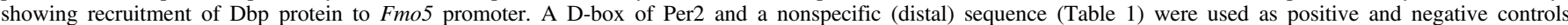
respectively. Data are mean \pm S.D. $(n=5)$. ${ }^{*} P<0.05$ ( $t$ test $)$. RLU: relative luciferase unit. 


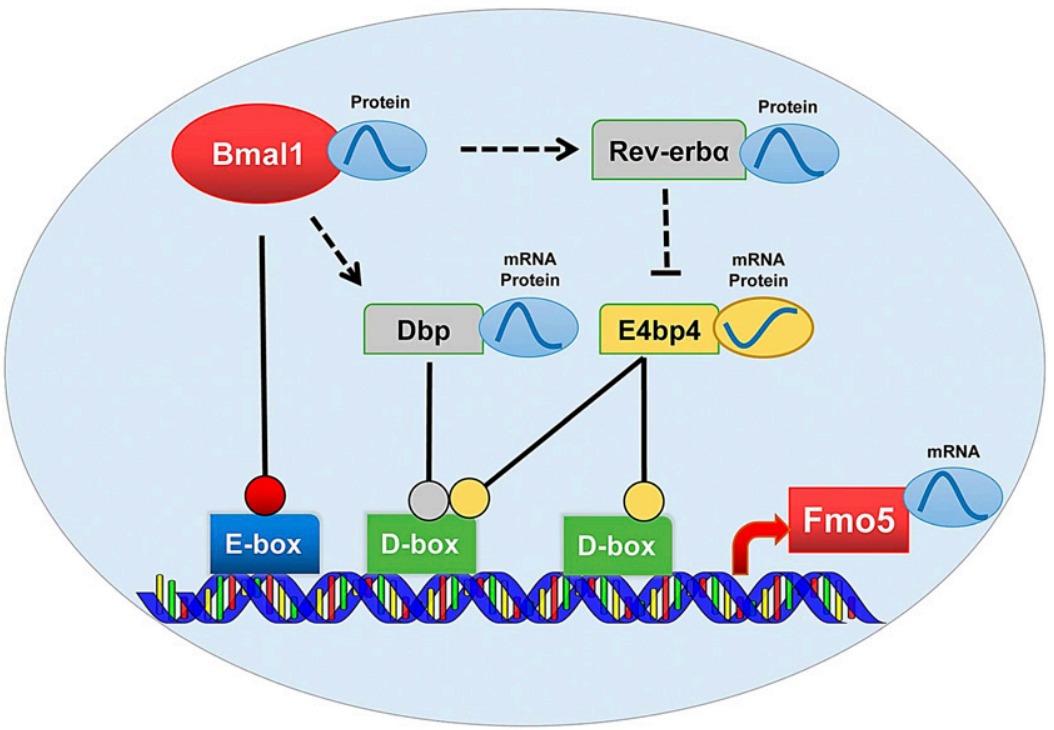

Fig. 8. Schematic diagram illustrating the molecular mechanism for generation of rhythmic Fmo5 expression. Fmo5 promoter presents E-box and D-box binding elements for transcriptional actions from circadian clock proteins such as Bmal1, E4bp4 and Dbp. In addition to direct transactivation, the E-box binding protein Bmall indirectly regulates the transcription of Fmo5 through Dbp and Rev-erb $\alpha / E 4 b p 4$ axis. Dashed arrows denote established regulatory pathways in the literature.

effects, including effects on fatty acid oxidation and biosynthesis of triglyceride, lipid, and cholesterol (Gonzalez Malagon et al., 2015), there is a possibility that this enzyme contributes to circadian hemostasis of endogenous substances.

We observed consistent diurnal patterns (peaks at the late light phase) between the regulator (Bmall1, Dbp, and Rev-erb $\alpha$ ) protein and Fmo5 mRNA, supporting positive transactivation of Fmo5 by these three factors (Fig. 8; Yu et al., 2019). Moreover, E4bp4 protein oscillated in antiphase to Fmo5 mRNA consistent with a negative regulation mechanism (Fig. 8; Yu et al., 2019). Our data lend support to the notion that Dbp and E4bp4 play an antagonistic role in gene regulation (Mitsui et al., 2001; Fig. 8). Dbp action (induction of Fmo5 expression) dominates in the daytime period, whereas E4bp4 action (inhibition of Fmo5 expression) dominates in the night, thereby contributing a diurnal oscillation in Fmo5 expression (Fig. 8).

Chronopharmacokinetic experiments with PTX revealed a more extensive formation of PTX-M (an Fmo5-dependent metabolite) at dosing time of ZT14 than at ZT2 consistent with the circadian time differences in Fmo5 expression (Figs. 1 and 2). However, the pharmacokinetic behavior of PTX was dosing time-independent, although slight differences in plasma/liver concentrations were observed (Fig. 2). This was probably because PTX-M formation by Fmo5 was a nonmajor metabolic pathway for PTX as P450 enzymes make a significant contribution (Peterson et al., 2004; Uney et al., 2019). Alterations in a nonmajor metabolic pathway may not cause sufficient changes in total parent drug metabolism and pharmacokinetics.

The current study and our previous studies support the notion that drug metabolism is under the control of the circadian clock (Zhang et al., 2018a; Zhao et al., 2018; Guo et al., 2018). Clock-controlled metabolism is associated with time-dependent pharmacokinetics and drug toxicity and effects (Johnson et al., 2014). We show herein that diurnal Fmo5 expression was regulated through the cis-acting elements E-box and D-box. A diurnal rhythm in FMO expression was also noted in rabbit lung (Hlavica and Damani, 2012). By using a computational algorithm (jaspar.genereg.net), we identified potential E-box and D-box elements in promoter regions of the other four Fmo genes (i.e., Fmo1, Fmo2, Fmo3, and Fmo4) (Supplementary Fig. S4). Therefore, it is highly probable that Fmol-4 are also circadian genes and are regulated by E-box and D-box binding proteins.
A serum shock experiment was performed to confirm the rhythmic expression of Fmo5 at a cellular level, because a serum shock (50\% serum) can induce circadian gene expression in the cells (Balsalobre et al., 1998). Mechanistically, a high level of serum activates serum response factor (SRF) to stimulate the expressions of target genes, including circadian clock genes Bmal1 and Per, inducing circadian gene expression (Gerber et al., 2013). Fmo5 mRNA appears to oscillate with a period of around 24 hours in serum-shocked cells (Fig. 1E). However, the amplitude was attenuated during the second period (Fig. 1E). This is normal because serum shock effect is timedependent, becoming weaker at a later stage (Murakami et al., 2008; Matsunaga et al., 2008; Yu et al.; 2019).

CLOCK is another E-box binding protein (Preitner et al., 2002). We attempted to validate the role of the E-box element in rhythmic regulation of Fmo5 using Clock $^{-1-}$ mice. Clock ablation led to downregulation of Fmo5 expression and loss of diurnal rhythm in mouse liver (Supplementary Fig. S5). The data strongly support a critical role for E-box transcription in generation of Fmo5 rhythmicity. It is noteworthy that an E-box ( $-581 /-575$ bp) and a D-box $(-600 /-589$ bp) can be found in promoter region of human FMO5 gene. There is a possibility that human FMO5 is a circadian gene and controlled by E-box and D-box binding proteins. However, whether this is true or not awaits further investigations.

In summary, we have shown diurnal rhythms of hepatic Fmo5 at the levels of mRNA, protein, and enzymatic activity. Fmo5 rhythms were generated through transcriptional actions of circadian clock genes (including Bmal1, E4bp4, and Dbp) on E-box and D-box ciselements. Our study has implications for understanding of clockcontrolled drug metabolism and for facilitating the practice of chronotherapeutics.

\section{Authorship Contributions \\ Participated in research design: Chen, Guan, Zhang, Wu. \\ Conducted experiments: Chen, Guan, $\mathrm{Xu}$. \\ Performed data analysis: Chen, Yu, Zhang, Wu. \\ Wrote or contributed to the writing of the manuscript: Chen, Wu.}

\section{References}

Balsalobre A, Damiola F, and Schibler U (1998) A serum shock induces circadian gene expression in mammalian tissue culture cells. Cell 93:929-937.

Cashman JR and Zhang J (2006) Human flavin-containing monooxygenases. Annu Rev Pharmacol Toxicol 46:65-100. 
Chen M, Guo L, Dong D, Yu F, Zhang T, and Wu B (2019) The nuclear receptor Shp regulates morphine withdrawal syndrome via modulation of Ugt2b expression in mice. Biochem Pharmacol 161:163-172.

Duez H, van der Veen JN, Duhem C, Pourcet B, Touvier T, Fontaine C, Derudas B, Baugé E, Havinga R, Bloks VW, et al. (2008) Regulation of bile acid synthesis by the nuclear receptor Rev-erbalpha. Gastroenterology 135:689-698.

Fiorentini F, Romero E, Fraaije MW, Faber K, Hall M, and Mattevi A (2017) Baeyer-Villiger monooxygenase FMO5 as entry point in drug metabolism. ACS Chem Biol 12:2379-2387.

Gachon F and Firsov D (2011) The role of circadian timing system on drug metabolism and detoxification. Expert Opin Drug Metab Toxicol 7:147-158.

Gekakis N, Staknis D, Nguyen HB, Davis FC, Wilsbacher LD, King DP, Takahashi JS, and Weitz CJ (1998) Role of the CLOCK protein in the mammalian circadian mechanism. Science $\mathbf{2 8 0}$ $1564-1569$.

Gerber A, Esnault C, Aubert G, Treisman R, Pralong F, and Schibler U (2013) Blood-borne circadian signal stimulates daily oscillations in actin dynamics and SRF activity. Cell $\mathbf{1 5 2}$ 492-503.

Gonzalez Malagon SG, Melidoni AN, Hernandez D, Omar BA, Houseman L, Veeravalli S, Scott F, Varshavi D, Everett J, Tsuchiya Y, et al. (2015) The phenotype of a knockout mouse identifies flavin-containing monooxygenase 5 (FMO5) as a regulator of metabolic ageing. Biochem Pharmacol 96:267-277.

Guo L, Yu F, Zhang T, and Wu B (2018) The clock protein Bmall regulates circadian expression and activity of sulfotransferase 1a1 in mice. Drug Metab Dispos 46:1403-1410.

Hernandez D, Janmohamed A, Chandan P, Phillips IR, and Shephard EA (2004) Organization and evolution of the flavin-containing monooxygenase genes of human and mouse: identification of novel gene and pseudogene clusters. Pharmacogenetics 14:117-130.

Hlavica P and Damani LA (2012) Factors regulating the activity of the rabbit lung flavin-containing mono-oxygenase, $\mathrm{N}$-Oxidation of Drugs: Biochemistry, pharmacology, toxicology, Springer Science \& Business Media.

Janmohamed A, Hernandez D, Phillips IR, and Shephard EA (2004) Cell-, tissue-, sex- and developmental stage-specific expression of mouse flavin-containing monooxygenases (Fmos). Biochem Pharmacol 68:73-83.

Johnson BP, Walisser JA, Liu Y, Shen AL, McDearmon EL, Moran SM, McIntosh BE, Vollrath AL, Schook AC, Takahashi JS, et al. (2014) Hepatocyte circadian clock controls acetaminophen bioactivation through NADPH-cytochrome P450 oxidoreductase. Proc Natl Acad Sci USA 111 18757-18762.

Kume K, Zylka MJ, Sriram S, Shearman LP, Weaver DR, Jin X, Maywood ES, Hastings MH, and Reppert SM (1999) mCRY1 and mCRY2 are essential components of the negative limb of the circadian clock feedback loop. Cell 98:193-205.

Krueger SK and Williams DE (2005) Mammalian flavin-containing monooxygenases: structure/ function, genetic polymorphisms and role in drug metabolism. Pharmacol Ther 106:357-387.

Lai WG, Farah N, Moniz GA, and Wong YN (2011) A Baeyer-Villiger oxidation specifically catalyzed by human flavin-containing monooxygenase 5. Drug Metab Dispos 39:61-70.

Lévi F, Okyar A, Dulong S, Innominato PF, and Clairambault J (2010) Circadian timing in cancer treatments. Annu Rev Pharmacol Toxicol 50:377-421.

Malátková P and Wsól V (2014) Carbonyl reduction pathways in drug metabolism. Drug Metab $\operatorname{Rev}$ 46:96-123.

Matsunaga N, Ikeda M, Takiguchi T, Koyanagi S, and Ohdo S (2008) The molecular mechanism regulating 24-hour rhythm of CYP2E1 expression in the mouse liver. Hepatology 48:240-251.

Matsunaga N, Inoue M, Kusunose N, Kakimoto K, Hamamura K, Hanada Y, Toi A, Yoshiyama Y, Sato F, Fujimoto K, et al. (2012) Time-dependent interaction between differentiated embryo chondrocyte- 2 and CCAAT/enhancer-binding protein $\alpha$ underlies the circadian expression of CYP2D6 in serum-shocked HepG2 cells. Mol Pharmacol 81:739-747.

Minami Y, Ode KL, and Ueda HR (2013) Mammalian circadian clock: the roles of transcriptional repression and delay. Handb Exp Pharmacol 217:359-377.

Mitsui S, Yamaguchi S, Matsuo T, Ishida Y, and Okamura H (2001) Antagonistic role of E4BP4 and PAR proteins in the circadian oscillatory mechanism. Genes Dev 15:995-1006.

Mukherji A, Kobiita A, Ye T, and Chambon P (2013) Homeostasis in intestinal epithelium is orchestrated by the circadian clock and microbiota cues transduced by TLRs. Cell 153:812-827.

Murakami Y, Higashi Y, Matsunaga N, Koyanagi S, and Ohdo S (2008) Circadian clock-controlled intestinal expression of the multidrug-resistance gene mdrla in mice. Gastroenterology 135 1636-1644.e3.
Nicklasson M, Björkman S, Roth B, Jönsson M, and Höglund P (2002) Stereoselective metabolism of pentoxifylline in vitro and in vivo in humans. Chirality 14:643-652.

Ohdo S, Koyanagi S, Suyama H, Higuchi S, and Aramaki H (2001) Changing the dosing schedule minimizes the disruptive effects of interferon on clock function. Nat Med 7:356-360

Ohmi N, Yoshida H, Endo H, Hasegawa M, Akimoto M, and Higuchi S (2003) S-oxidation of S-methyl-esonarimod by flavin-containing monooxygenases in human liver microsomes. Xenobiotica 33:1221-1231.

Paschos GK, Baggs JE, Hogenesch JB, and FitzGerald GA (2010) The role of clock genes in pharmacology. Annu Rev Pharmacol Toxicol 50:187-214

Peterson TC, Peterson MR, Wornell PA, Blanchard MG, and Gonzalez FJ (2004) Role of CYP1A2 and CYP2E1 in the pentoxifylline ciprofloxacin drug interaction. Biochem Pharmacol 68: $395-402$.

Preitner N, Damiola F, Lopez-Molina L, Zakany J, Duboule D, Albrecht U, and Schibler U (2002) The orphan nuclear receptor REV-ERBalpha controls circadian transcription within the positive limb of the mammalian circadian oscillator. Cell 110:251-260.

Ralph MR, Foster RG, Davis FC, and Menaker M (1990) Transplanted suprachiasmatic nucleus determines circadian period. Science 247:975-978.

Raoul JM, Peterson MR, and Peterson TC (2007) A novel drug interaction between the quinolone antibiotic ciprofloxacin and a chiral metabolite of pentoxifylline. Biochem Pharmacol 74: 639-646.

Rendic S and Guengerich FP (2015) Survey of human oxidoreductases and cytochrome P450 enzymes involved in the metabolism of xenobiotic and natural chemicals. Chem Res Toxicol 28: $38-42$.

Reppert SM and Weaver DR (2002) Coordination of circadian timing in mammals. Nature 418 935-941.

Samlaska CP and Winfield EA (1994) Pentoxifylline. J Am Acad Dermatol 30:603-621

Uney K, Tras B, Corum O, Yildiz R, and Maden M (2019) Pharmacokinetics of pentoxifylline and its 5-hydroxyhexyl metabolite following intravenous administration in cattle. Trop Anim Health Prod 51:435-441.

Wang S, Lin Y, Yuan X, Li F, Guo L, and Wu B (2018) REV-ERB $\alpha$ integrates colon clock with experimental colitis through regulation of NF-кB/NLRP3 axis. Nat Commun 9:4246.

Yamaguchi S, Mitsui S, Yan L, Yagita K, Miyake S, and Okamura H (2000) Role of DBP in the circadian oscillatory mechanism. Mol Cell Biol 20:4773-4781.

Yin L and Lazar MA (2005) The orphan nuclear receptor Rev-erbalpha recruits the N-CoR/ histone deacetylase 3 corepressor to regulate the circadian Bmal1 gene. Mol Endocrinol 19 $1452-1459$

Yu F, Zhang T, Zhou C, Xu H, Guo L, Chen M, and Wu B (2019) The circadian clock gene Bmall controls intestinal exporter MRP2 and drug disposition. Theranostics 9:2754-2767.

Zhang T, Guo L, Yu F, Chen M, and Wu B (2019) The nuclear receptor Rev-erb $\alpha$ participates in circadian regulation of Ugt2b enzymes in mice. Biochem Pharmacol 161:89-97.

Zhang T, Yu F, Guo L, Chen M, Yuan X, and Wu B (2018a) Small heterodimer partner regulates circadian cytochromes p450 and drug-induced hepatotoxicity. Theranostics 8:5246-5258.

Zhang T, Zhao M, Lu D, Wang S, Yu F, Guo L, Wen S, and Wu B (2018b) REV-ERB $\alpha$ regulates CYP7A1 through repression of liver receptor homolog-1. Drug Metab Dispos 46:248-258.

Zhang W, Sargis RM, Volden PA, Carmean CM, Sun XJ, and Brady MJ (2012) PCB 126 and other dioxin-like PCBs specifically suppress hepatic PEPCK expression via the aryl hydrocarbon receptor. PLoS One 7:e37103.

Zhang YK, Yeager RL, and Klaassen CD (2009) Circadian expression profiles of drug-processing genes and transcription factors in mouse liver. Drug Metab Dispos 37:106-115.

Zhao M, Zhang T, Yu F, Guo L, and Wu B (2018) E4bp4 regulates carboxylesterase 2 enzymes through repression of the nuclear receptor Rev-erb $\alpha$ in mice. Biochem Pharmaco 152:293-301.

Address correspondence to: Dr. Baojian Wu, College of Pharmacy, Jinan University, 601 Huangpu Avenue West, Guangzhou 510632, China. E-mail: bj.wu@ hotmail.com; or Dr. Tianpeng Zhang, College of Pharmacy, Jinan University, 601 Huangpu Avenue West, Guangzhou 510632, China. E-mail: tp.zhang@outlook.com 